\title{
Reflections from the 2019 International Cancer Education Conference in Salt Lake City, Utah
}

\author{
Ewa Szumacher ${ }^{1}$
}

Published online: 12 November 2019

(C) American Association for Cancer Education 2019

This year's International Cancer Education Conference, the prime meeting in cancer education, took place in Salt Lake City, Utah, in the Little America Hotel.

Salt Lake City is more than an urban center; it is also a place of great natural beauty with the stunning Wasatch Mountain Range to the east and the shimmering blue waters of the Great Salt Lake to the west. We thank our colleagues in Salt Lake City for hosting us in this exceptionally beautiful part of the continent.

The theme of this year's conference was "Revolutionizing Cancer Education Globally through Technology across Generations." In addition to putting together an excellent scientific program, the planning committee of the 2019 ICEC also organized a very engaging social itinerary, which introduced the conference attendees to Salt Lake City's numerous fascinating historical, religious, and cultural landmarks.

The International Cancer Education Conference is organized in partnership with the American Association for Cancer Education, the Cancer Patient Education Network and the European Association for Cancer Education. The purpose of the event is to support cancer educators in learning best practices for cancer prevention, diagnosis, treatment, and survivorship. The specific goal of this year's conference was to discuss new and creative models that support best practice developments in the field and to facilitate interdisciplinary research and collaboration across the cancer care continuum on a national and international level.

As every year, several engaging and informative preconference workshops were presented. The "NCI R25 training workshop supporting cancer education projects through the NCI R25 Grant program,” presented by Jeannette Korczak,

Ewa Szumacher

ewa.szumacher@sunnybrook.ca

1 Sunnybrook Odette Cancer Centre, Rm T2-150, 2075 Bayview Avenue, T-Wing, Toronto, ON M4N3M5, Canada
Kathleen Blazer, Jeffrey Wetzel, Amr Soliman, and Robert Chamberlain, provided useful and practical tools to facilitate successful NCI R25 grant writing. The workshop stimulated constructive discussions among participants regarding the process of NCI R25 grant preparations and generated many useful tips and advice. Another worthwhile workshop, "The Art of Journal Writing and Reviewing: Key to Success," presented by Maria Bishop, John Vetto, Jill Hamilton, and Darren Starmer, offered practical and actionable advice for authors on writing a publishable manuscript, and for reviewers about how to effectively peer review manuscripts. In line with the conference theme, Daniel Cronin presented on empowering cancer educators through optimizing video technology for teaching. He discussed three key principles of effective educational videos, which would allow participants to broadly disseminate their clinical knowledge and expertise. Continuing on the theme of communication, Cathy Meade, Shannon Christy, and Clement Guide emphasized the importance of considering culture and health literacy in cancer communications and how best to incorporate modern technology in cancer-care communications. Finally, "Concept Mapping in Cancer Education and Care Planning" presented by Miklos Fograsi was very well received by the workshop attendees who learned how to concept map and how concept mapping could be used to simplify complex medical topics for patients and multidisciplinary teams.

In the outstanding opening plenary, Mary C Beckerle, CEO of the Huntsman Cancer Institute, provided a perspective on cancer patient-centered care in Salt Lake City and the state of Utah, and highlighted the major technological innovations in teaching, research, and clinical care at the Huntsman Cancer Institute. Specifically, Dr. Beckerle's comprehensive overview of education projects geared at various generations and social groups offered participants a broad range of useful approaches to cancer education for varying audiences, including pamphlets, presentations, social media, videos, and telehealth. Additionally, conference participants had the opportunity to visit this modern state-of-the-art cancer center during the tour 
and the welcome reception. We thank the local organization committee for coordinating this enlightening visit.

The highlight of the conference was the exceptional keynote speakers. On Thursday morning, David Wiljer from the University Health Network in Toronto and past president of the American Association for Cancer Education, presented "Emerging Technologies and Digital Compassion: Embracing a Grand Challenge for Cancer Education." Dr. Wiljer's excellent talk provided some captivating thoughts on the impact of e-health technologies in cancer care: technology will increase the potential for patient engagement and activation, and change the nature of the therapeutic relationship between patients and health care providers.

Later on, Sabine Fromm-Haidenberger, the current president of the European Association Cancer Education (EACE), gave a very personal, informative, and reflective talk about developments in cancer education within EACE over the past decade. She highlighted the areas for potential close collaboration between AACE and EACE, such as the development of a mentorship program, leadership in cancer education, and other areas that can be addressed at the EACE and AACE annual meetings.

This year's Samuel C. Harvey lecture entitled "An Ethics Lens on Education about Cancer or Cancer Education" was delivered by Reuben C Warren. Dr. Warren is currently the Director of the National Center for Bioethics in Research and Health Care and Professor of Bioethics at Tuskegee University in Tuskegee, Alabama. Dr. Warren helped the conference audience to understand how bioethics relates to everyday life, including learning from a difficult past, looking at current issues related to racial disparities in disease and treatment, and showing how we can be more empowered as healthcare consumers, as well as become more informed research participants.

The patient panel discussed informational needs of individuals with inherited cancer predisposition. The panel, presented by three women with inherited cancer, helped us to better understand the life challenges of patients genetically predisposed to cancer. The discussion explored aspects of social, emotional, and physical adaptation to diagnoses, screening, and prophylactic treatment that affect patients with inherited cancer.

The last keynote speech, presented by Ken R Smith from the Huntsman Cancer Institute, discussed the unique and comprehensive Family Cancer Histories and The Utah Population Database, which is a stellar example of how databases can be used to benefit cancer prevention and screening.

There were other highlights at this year's AACE conference as well. The poster sessions included 82 posters, which generated many productive and meaningful interactions between the conference participants. Often, student-presented posters facilitate connections between these students and their future mentors, thus continuing the inter-generational passing of knowledge and advancing the discipline.

The social itinerary of the conference was outstanding. Participants thoroughly enjoyed the conference dinner in Temple Square, located in the Empire Room at the Joseph Smith Memorial Building. The conference attendees were able to choose to visit the Family History Library at Temple Square, which contains the largest genealogical library in the world, or to tour the Temple Square Conference Center, including artwork and rooftop gardens. Both options were well-received and provided participants with a charming glimpse into the local history and culture.

The ICEC annual meeting was a resounding success. The conference facilitated many productive discussions, stimulated collaborations, and shared best practices among the 250 attendees. In addition to the informative and thoughtprovoking talks and sessions, we recognized many of our distinguished colleagues for their contributions to cancer education through awards and other recognitions. As in previous meetings, the atmosphere of the conference was found to be excellent by all who participated; the scientific sessions, workshops, and social activities promoted collegial and scientifically productive dialogue which we hope to continue and expand through ongoing collaborations throughout the year. We are grateful to all organizers and participants and look forward to meeting again next year in College Park, Maryland. 